\title{
Moderne Gesellschaften brauchen eine aktive Dienstleistungspolitik
}

Der wirtschaftliche und gesellschaftliche Strukturwandel zeigt - trotz der nach wie vor unstrittigen Bedeutung des industriellen Sektors, besonders in Deutschland, - im langfristigen Trend eine deutliche Verschiebung der Beschäftigungsanteile: von der Industrie zu den Dienstleistungen. Jedoch sind unterschiedliche Wege - „high roads“ und „low roads“ - möglich. Derzeitige fragwürdige politische Weichenstellungen verweisen auf Letzteres und bergen das Risikovon Beschäftigungs- und Wachstumsverlusten im Dienstleistungsbereich. Angesichts der zunehmenden gesellschaftlichen wie auch der gesamtwirtschaftlichen Bedeutung von Dienstleistungen betont der Beitrag die Rolle des Staates bei der Entwicklung gesellschaftlich notwendiger Dienstleistungen und diskutiert unterschiedliche Tertiarisierungswege sowie ihre Implikationen. Dabei schält sich heraus: Die Nachfrageseite muss eine tragende Rolle spielen, wenn Arbeitslosigkeit, prekäre Beschäftigung und gesellschaftliche Spaltung verhindert werden sollen.

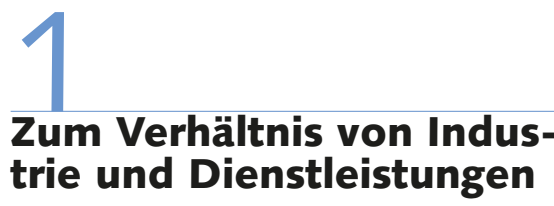

Die unerwartet schnelle wirtschaftliche Erholung Deutschlands nach dem krisenbedingen Produktionsrückgang beruhte vornehmlich auf dem Güterexport. Somit fand die jüngste Wachstumsbelebung primär im industriellen Bereich statt. Im Unterschied zu den starken Produktions- und Beschäftigungsschwankungen der industriellen Produktion und der ihr unmittelbar verbundenen Dienstleistungen weisen die haushaltsorientierten sowie die öffentlichen Dienstleistungen deutlich geringere Schwankungen auf. Dieser Sachverhalt gilt nicht nur für Deutschland, sondern findet sich auch in anderen vergleichbaren Volkswirtschaften. Daraus lässt sich die These herleiten, dass der industrielle Bereich zwar deutlicher auf wirtschaftliche Krisen und Abschwünge reagiert, aber kurzfristig auch stärker zum Wachstum beiträgt. Haushaltsbezogene und öffentliche Dienstleistungen sind auf kurze Sicht eher wachstumsschwach. Auflängere Sicht ändert sich jedoch das Bild. Der Anteil industrieller Beschäftigung sinkt und Beschäftigungszuwachs findet fast nur noch bei den Dienstleistungen statt (Werner 2001, S. 289), sodass sie in erheblichem Umfang sowohl die Beschäftigung als auch die gesamtwirtschaftliche Wertschöpfung stabilisieren.

Vor diesem Hintergrund stellt sich die Frage, ob die in der Vergangenheit weitgehend unbestrittene These der Notwendig- keit einer stärkeren Ausrichtung auf den Dienstleistungssektor mit Blick auf die jüngere Vergangenheit zu überdenken ist, wie dies etwa vom Bundeswirtschaftsministerium propagiert wird (BMWi 2010, S. 6). Ist eine Re-Konzentration auf die Industriepolitik tatsächlich die gebotene Antwort auf die aktuellen wirtschaftlichen, sozialen und ökologischen Anforderungen der Gegenwart? Oder sollte nicht vielmehr die Förderung binnenmarktorientierter Dienstleistungen, die dem gesellschaftlichen Bedarf entsprechen, gerade wegen ihrer hervorstechenden gesamtwirtschaftlichen Stabilisierungsfunktion im Vordergrund stehen? Dies auch deswegen, weil sich höchstwahrscheinlich der relative Anteilsverlust industrieller Beschäftigung infolge der hohen Rationalisierungspotenz industrieller Produktion auch künftig fortsetzt, was gleichzeitig zur Aufrechterhaltung bzw. Stärkung der industriellen Wettbewerbsfähigkeit notwendig und erwünscht ist. Vor diesem Hintergrund käme einer die industriellen Beschäftigungsverluste kompensierenden Expansion der ausbaufähigen und dem gesellschaftlichen Bedarf entsprechenden Dienstleistungsbereiche eine hohe Bedeutung zu.

Um vorstehende Fragen eingehender beantworten zu können, wird nach einem empirischen Überblick über den langfristigen strukturellen Wandel (Abschnitt 2) zunächst auf das (alte) Problem der Definition von Dienstleistungen eingegangen (Abschnitt 3). Im Anschluss werden die langfristigen Triebkräfte der wirtschaftlichen Entwicklung und die hiermit verbundenen Strukturveränderungen darge- legt (Abschnitt 4). Auf dieser Grundlage können dann wirtschafts- und beschäftigungspolitische Handlungsalternativen aufgezeigt (Abschnitt 5) und vor allem Inhalte einer erfolgreichen Wirtschaftsund Beschäftigungspolitik (Abschnitt 6) diskutiert werden. Abschnitt 7 resümiert die politischen Handlungsanforderungen.

\section{2 \\ Von der Agrar- zur Dienst- leistungswirtschaft}

Der Rückblick auf die langfristige Veränderung der gesamtwirtschaftlichen Struktur lässt klar erkennen, dass alle Ökonomien mit intensivem Wachstum einem weitge-

Norbert Reuter, PD Dr., ist Ökonom im Bereich Wirtschaftspolitik beim Bundesvorstand der Vereinten Dienstleistungsgewerkschaft (ver.di), lehrt Volkswirtschaftslehre an der Rheinisch-Westfälischen Technischen Hochschule (RWTH) Aachen und ist sachverständiges Mitglied der Enquete-Kommission "Wachstum, Wohlstand, Lebensqualität" des Deutschen Bundestages. Arbeitsschwerpunkte: Strukturwandel, Verteilung, demografische Entwicklung. e-mail: norbert.reuter@verdi.de Karl Georg Zinn, Prof. Dr., ist emeritierter Hochschullehrer der Volkswirtschaftslehre an der RWTH Aachen.

Arbeitsschwerpunkte: Geschichte der Politischen Ökonomie, sozialökonomische Krisen, Postwachstumsökonomie(n). e-mail: Zinn-Wiesbaden@t-online.de 
hend gleichen längerfristigen Trend des Strukturwandels unterliegen: von der Dominanz des Agrarsektors über die starke Ausweitung des industriellen Sektors hin zur überwiegenden Dienstleistungsproduktion. Diese Regelmäßigkeit in der historischen Entwicklung der Wirtschaftsstruktur hat dazu geführt, die drei Sektoren gemäß der Veränderung ihrer relativen Bedeutung im Zeitverlauf als „primären“, „sekundären“ und „tertiären“ Sektor zu bezeichnen, was sich auch in der bis heute üblichen statistischen Zuordnung von Branchen zu den drei Sektoren widerspiegelt. Die historische Gewichtsverschiebung der Sektoren lässt sich sowohl an der relativen Zahl der Beschäftigten als auch an dem Wertschöpfungsanteil eines Sektors erkennen (Reuter 2010, S. 86ff.).

1950 war nach der Klassifikation des Statistischen Bundesamtes noch rund ein Viertel aller Beschäftigten in Deutschland im primären Sektor ${ }^{1}$ tätig. Seitdem ging sein Anteil beständig bis auf heute nur noch gut $2 \%$ zurück. Bis 2025 prognostiziert das Institut für Arbeitsmarkt- und Berufsforschung (IAB) einen weiteren Rückgang der Beschäftigung im primären Sektor auf 1,5\% (Fuchs/Zika 2010, S. 5; Abbildung 1).

Der sekundäre Sektor ${ }^{2}$ expandierte bis Ende der 1960er Jahre. Zu dieser Zeit waren hier knapp $50 \%$ aller Erwerbstätigen beschäftigt. Seitdem nimmt der Anteil dieses Sektors an der Gesamtbeschäftigung wie an der Wertschöpfung kontinuierlich ab. 2025 wird nach den Schätzungen des IAB nur noch gut ein Fünftel der Erwerbstätigen in diesem Sektor beschäftigt sein (ebd.).

Parallel, allerdings auf niedrigerem Niveau und zunächst deutlich langsamer, expandierte der tertiäre bzw. der Dienstleistungssektor ${ }^{3}$. Anfang der 1970er Jahre überholte er aber sowohl hinsichtlich der Wertschöpfung als auch der Beschäftigung den sekundären Sektor. Seit 1972 arbeitet in Deutschland die Mehrheit der Erwerbstätigen in Branchen, die dem tertiären Sektor zugerechnet werden. 2025 werden laut IAB knapp $77 \%$ aller Erwerbstätigen mit Dienstleistungen beschäftigt sein (Abbildung 1).

Doch trotz der stark gestiegenen Bedeutung von Dienstleistungen zeigt der internationale Vergleich, dass Deutschland hier gegenüber Ländern mit ähnlich hohem Pro-Kopf-Einkommen - insbesondere gegenüber den skandinavischen

\section{Abb. 1: Entwicklung der Erwerbstätigkeit in Deutschland}

in den klassischen drei Sektoren - Anteile in \% -

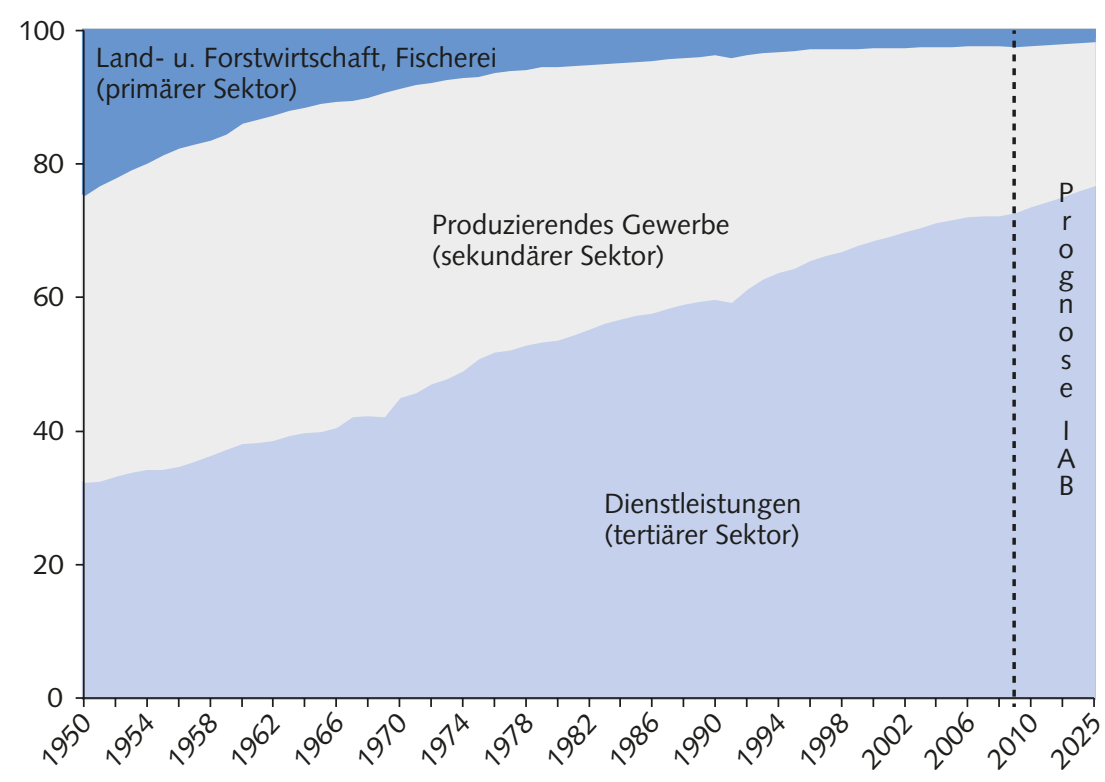

Quelle: Daten bis 2009: Statistisches Bundesamt, Volkswirtschaftliche Gesamtrechnung; bis 1990 früheres Bundesgebiet einschl. Berlin-West. Daten für 2025: Prognose des Instituts für Arbeitsmarkt- und Berufsforschung (IAB); Daten 2009-2025 interpoliert

\section{WSI MITTEILUNGEN}

Ländern - deutlich zurückliegt und noch einen vergleichsweise großen Industriesektor aufweist. Entgegen aller Trends ist sein Wertschöpfungsanteil - im Unterschied zu seinem Beschäftigtenanteil - seit 2004 zeitweise sogar wieder leicht gestiegen. Wesentliche Ursache hierfür ist die hohe Exportorientierung der deutschen Wirtschaft, die durch die anhaltende reale Stagnation der Arbeitseinkommen befördert wurde (Joebges et al. 2009, S. 7f.). Kehrseite ist eine ausgeprägte Schwäche der Binnennachfrage mit negativen Folgen für den privaten Konsum im Allgemeinen und die Nachfrage nach heimischen Dienstleistungen im Besonderen (Horn et al. 2011, S. 22).

\section{3 \\ Drei Sektoren, aber eine Volkswirtschaft - Struktur und Interdependenzen}

Die von der amtlichen Statistik benutzte Aufteilung der Wirtschaft in drei Sektoren spiegelt die grundsätzliche Tendenz der Entwicklung treffend wider. Theoretisch überzeugende und praktikable Alternativen, die den gesamtwirtschaftlichen Strukturwandel statistisch detaillierter abbilden können, liegen auch nicht vor.
Solange dies nicht der Fall ist, wird man auf die Einteilung nach Sektoren nicht verzichten können.

Grundsätzlich wäre es jedoch wünschenswert, in der statistischen Erfassung der Wirtschaftsstruktur der Tatsache Rechnung zu tragen, dass alle Güter, also auch Dienstleistungen, unter Berücksichtigung der Vorleistungen in der Regel „zusammengesetzte Güter" (composite goods) sind (Schettkat 2010, S. 10ff.). Deutlich wird das an den einzelnen Kostenkomponenten einer Dienstleistung. Beispielsweise sind im Tagespreis eines Mietwagens anteilig auch die Anschaffungskosten des Fahrzeugs enthalten. Die Ausweitung einer Dienstleistungsproduktion im engeren Sinne ist deshalb fast immer mit einer

\footnotetext{
1 Land- und Forstwirtschaft, Fischerei und Fischzucht.

2 Verarbeitendes Gewerbe, Energie- und Wasserversorgung, Baugewerbe, Bergbau und Gewinnung von Steinen und Erden.

3 Handel, Instandhaltung und Reparatur von $\mathrm{Kfz}$ und Gebrauchsgütern, Gastgewerbe, Verkehr- und Nachrichtenübermittlung, Kredit- und Versicherungsgewerbe, Grundstückswesen, Vermietung, unternehmensbezogene Dienstleistungen, öffentliche Verwaltung, Verteidigung, Sozialversicherungen, Erziehung und Unterricht, Gesundheits-, Veterinär- und Sozialwesen, sonstige öffentliche und private Dienstleister, häusliche Dienste.
} 
mehr oder weniger großen Produktionszunahme der der Dienstleistung inkorporierten bzw. der zu ihr komplementären Industriegüter verbunden, was etwa auch im Begriff der "hybriden Wertschöpfung“ seinen Ausdruck findet.

Güter, also auch Dienstleistungen, als „zusammengesetzt“ im Sinn der „composite goods“ zu verstehen, hat Konsequenzen für die Untersuchung des strukturellen Wandels. Denn mit voranschreitendem Strukturwandel wird das relative Gewicht der einzelnen Komponenten eines zusammengesetzten Gutes verändert. Da letztlich alle produzierten Güter auf den Einsatz der beiden originären Produktionsfaktoren Arbeit und Natur zurückgeführt werden können - Sachkapital ist bekanntlich kein originärer Faktor, sondern selbst auf Arbeit und natürliche Ressourcen reduzierbar -, bietet es sich an, zwischen lebendiger und vergegenständlichter Arbeit zu unterscheiden. Der Strukturwandel schlägt sich so gesehen in Anteilsverschiebungen von vergegenständlichter zu lebendiger Arbeit in den zusammengesetzten Gütern nieder. Bei den Dienstleistungen im herkömmlichen Sinn ergeben sich natürlich erhebliche Unterschiede bezüglich des Vorleistungsanteils „vergegenständlichte Arbeit“. Er dürfte relativ hoch bei der Krankenhausdienstleistung sein, hingegen relativ niedrig bei Kinderbetreuung, mobilen Pflegediensten und dergleichen, ganz unabhängig davon, ob die Dienste privat oder von der öffentlichen Hand bereitgestellt werden.

Der landläufig als Tertiarisierung oder Entwicklung zur Dienstleistungsgesellschaft bezeichnete Strukturwandel wird zwar als makroökonomisches Phänomen wahrgenommen, aber selbstverständlich handelt es sich um die Aggregation der auf der mikroökonomischen Ebene ablaufenden strukturellen Veränderungen. Wenn die langfristige Entwicklung makroökonomisch betrachtet einen Strukturwandel zugunsten von Dienstleistungen erkennen lässt, so muss das auch in den mikroökonomischen Einheiten, bei den Unternehmen, sichtbar werden. In der Tat spielt etwa lebendige Arbeit als Vor- und zunehmend auch als Nachleistung der Sachgüterproduktion in den technischwirtschaftlich höchst entwickelten Volkswirtschaften eine immer bedeutendere Rolle. Es zeigt sich sowohl eine steigende wissenschaftlich-technische Abhängigkeit des industriellen Expansionspotenzials von der Ausweitung der Dienstleistungsproduktion (etwa die lebendige Arbeit in Forschungsinstituten) als auch ein zunehmender Rückgriff gerade anspruchsvoller Dienstleistungen auf industrielle Vorleistungen (z.B. Computerisierung von Arztpraxen). Wenn mehr und mehr Dienstleistungen als unverzichtbare Vorleistung für industrielle Innovation und industrielles Wachstum erforderlich sind, dann findet eine Art „Tertiarisierung“ im Rahmen der Industrie statt (Zinn 1998, S. 116ff.). Gleichwohl schlägt sich dies statistisch einzig in einer Ausweitung der Dienstleistungsbeschäftigung und damit des Dienstleistungssektors nieder.

Neben der vorstehend skizzierten quasi technischen Komplementarität ist die gegenseitige Abhängigkeit der Sektoren als Konsumenten-Kaufkraft bzw. Konsumnachfrage schaffende Aggregate $\mathrm{zu}$ betonen. Industriegüter werden zwar von allen Konsumenten nachgefragt, aber wenn die Beschäftigung und damit die Einkommenssumme in einem Sektor relativ oder sogar absolut sinkt, in einem anderen steigt, so kann die gesamtwirtschaftliche Konsumgüternachfrage nur erhalten bleiben und zunehmen, wenn ein entsprechend wachsender Anteil auf die Einkommensempfänger des wachsenden Sektors entfällt. Kurz gesagt, würde die Kfz-Industrie nur an Erwerbspersonen des sekundären Sektors verkaufen (können), so wäre es um diese Branche schlecht bestellt.

Die gesamtwirtschaftliche Bedeutung des nachfrageseitigen Aspekts des Strukturwandels ließ sich bereits beim Übergang von der Agrar- zur Industriegesellschaft erkennen. Denn der Absatz von Agrargütern war in steigendem Maße auf die Nachfrage aus dem industriellen Sektor angewiesen. Ohne einen solchen Nachfragesog hätten sich viele agrarwirtschaftliche Innovationen kaum gelohnt und wären zum Schaden von Wachstum und Wohlstand unterblieben. Gleiches gilt heute für das Verhältnis von sekundärem und tertiärem Sektor. Entwickelt sich die Nachfrage aus den tertiären Bereichen zu schwach - sei es wegen unzureichender Expansion tertiärer, u.a. öffentlicher Leistungen, sei es wegen eines weit hinter dem gesamtwirtschaftlichen Produktivitätswachstum zurückbleibenden Lohnniveaus - bleibt auch das Wachstums- und gegebenenfalls sogar das Innovationspotenzial ${ }^{4}$ des sekundären Sektors ungenutzt.

\section{4 \\ Ursachen des Sektorenwandels}

Aufbauend auf den Arbeiten von Allan Fisher (1935) und Colin Clark (1940) hatte Jean Fourastié (1949/1969) eine Theorie des langfristigen Strukturwandels vorgelegt. Die von Fourastié beschriebenen Triebkräfte des Sektorenwandels machen deutlich, welche Bedingungen und prinzipiellen Möglichkeiten für eine Anteilsstabilisierung oder gar das Wiedererstarken des industriellen Sektors vorliegen müssten. Seine Analyse erlaubt damit auch ein Urteil darüber, ob es überhaupt realistisch ist, diese Bedingungen wiederherzustellen.

Als Ursache des langfristigen Sektorenwandels sah Fourastié zwei Triebkräfte: den Produktivitätsfortschritt und relative Marktsättigungstendenzen. Der Produktivitätsfortschritt, also die technische und organisatorische Entwicklung, ermöglicht eine immer größere Produktion pro Arbeitseinheit. Allerdings erklärt dies alleine noch keinen Sektorenwandel. Solange sich das Mehrprodukt als absetzbar erweist, folgt aus dem Produktivitätsfortschritt lediglich eine fortgesetzte Expansion des betreffenden Sektors. Erst zusammen mit dem zweiten Faktor, der im Vergleich zum steigenden Angebot einsetzenden relativen Marktsättigung, kommt es wegen zunehmender Überkapazitäten zu Absatzproblemen und in der Folge zu Beschäftigungsverlusten in diesem Sektor.

Wegen der großen Nachfrage nach industriellen Gütern konnte der industrielle Sektor zunächst Arbeitskräfte, die im primären Sektor nicht mehr unterkamen, auffangen. Entsprechend expandierte der sekundäre Sektor. Da Fourastié auch in diesem Sektor die beiden Triebkräfte Produktivitätsfortschritt und Marktsättigung wirken sah, prognostizierte er bereits 1949 eine analoge Entwicklung wie beim Übergang vom primären zum sekundären Sektor: zunächst eine Ausdehnung dieses Sektors verbunden mit einer steigenden Produktion von industriell erzeugten

\footnotetext{
4 Je ungünstiger die Absatzerwartungen ausfallen desto riskanter erscheinen die für grundlegende Innovationen erforderlichen Investitionen. Dieser Nachfrageaspekt der Innovationstätigkeit wird von den einseitig auf die Angebotsseite gerichteten Innovationserklärungen ignoriert.
} 
Gütern. Früher oder später würde das wachsende Angebot aber auch hier auf eine weniger stark steigende bzw. dahinter zurückbleibende Nachfrage stoßen. Beschäftigungsverluste im industriellen Bereich würden nun durch Beschäftigungsgewinne im expandierenden tertiären Sektor, also im Dienstleistungsbereich, ausgeglichen.

Fourastiés „große Hoffnung des 20. Jahrhunderts" beruhte auf der Einschätzung, Dienstleistungen unterlägen im Unterschied zu den Produkten des primären und sekundären Sektors keinem oder nur einem vergleichsweise geringen Produktivitätsfortschritt. Zudem unterstellte er einen unstillbaren „Hunger nach Tertiärem“, also ein Ausbleiben von Marktsättigung in Bezug auf Dienstleistungen. Fourastié begründete dies damit, dass „der tertiäre Sektor entweder das Leben des Konsumenten sehr angenehm gestaltet (Theater, Schauspiel, Kunst, Tourismus, Dienstleistungen im Handelsverkehr) oder er [...] für die Gemeinschaft und sogar für die Herstellung von Produkten des primären und sekundären Sektors (Bildungswesen, Forschung, Verwaltung usw.) absolut unerläßlich ist" (Fourastié 1967, S. 29). Unbegrenzter Bedarf nach Dienstleistungen - als Endprodukte wie als zunehmende komplementäre Leistungen für die Aufrechterhaltung der industriellen Produktion im Sinne der „composite goods" - waren also die Bausteine seines optimistischen Zukunftsmodells.

Für den Fall, dass sich Dienstleistungen doch als stark rationalisierbar erweisen sollten, hielt es Fourastié nicht nur für möglich, sondern für geboten, die Staatsquote und damit den Kollektivkonsum (Produktion öffentlicher Güter) schneller anzuheben, als es sich ohnehin infolge der strukturellen Bedarfsverschiebungen fortgeschrittener Volkswirtschaften ergäbe. Ergänzend könnten dann auch verstärkt Arbeitszeitverkürzungen erfolgen (vgl. Reuter 2000, S. 190ff.).

Heute wissen wir, dass der langfristige Strukturwandel die theoretisch begründeten Voraussagen zu den Anteilsverschiebungen zwischen den drei Sektoren und den ihnen zugeordneten Tätigkeiten bestätigt. Jedoch kam es nicht zu einem reibungslosen, sozusagen "harmonischen“ Strukturwandel in Richtung Dienstleistungsgesellschaft. Für diese Fehlentwicklung sind falsche wirtschaftspolitische Weichenstellungen verantwortlich, zu denen auch die vermeintlich theoretisch begründbare Abwertung von Dienstleistungen als „Kostgänger“ der Industrie beigetragen hat.

Die „Kostgänger-These“ reproduziert einen bis auf die klassischen Ökonomen und die Physiokraten zurückgehenden Irrtum, dass bestimmte Produktionen bzw. die entsprechenden Arbeitsleistungen sozusagen auf Kosten anderer, quasi „fundamentaler" Güterproduktionen basierten. Gerade der staatliche Bereich wird oftmals als Kostgänger der Privatwirtschaft charakterisiert bzw. vielfach werden Dienstleistungen generell - zumindest ein Teil der Dienstleistungen - als Kostgänger der materiellen Produktion eingestuft.

Tatsächlich ignoriert die „KostgängerThese“ die vielfältigen sektoralen Interdependenzen und gegenseitigen Abhängigkeiten der einzelnen Sachgüter- und Dienstleistungsproduktionen - handele es sich um private oder öffentliche. Eine ganz andere Frage wäre die nach der relativen Wichtigkeit von Produktionen bzw. Gütern. Hier würde die Antwort gegebenenfalls unangenehme Überraschungen für manche Bereiche bringen, die sich als „fundamentale“ verstehen. Kurz gesagt, die Meinung, es gäbe Produktionen, die sich als „Kostgänger“ anderer, vermeintlich fundamentaler Produktionen charakterisieren ließen, beruht auf der Verwechslung von relativer Wichtigkeit einer Produktion mit dem Problem einer ausgewogenen Proportionierung der verschiedenen Branchen und Sektoren. Gleichgültig, in welchem Produktionsbereich einzelne ihr Einkommen verdienen, sie verbrauchen Güter und Dienstleistungen entsprechend dem üblichen Warenkorb (ihrer Einkommensschicht). Sie fragen Industriegüter und private sowie öffentliche Dienstleistungen entsprechend ihren Bedürfnissen nach, was aber nichts darüber aussagt, ob Lebensmittel, Volkshochschulkurse, Autoreifen oder medizinische Dienstleistungen für den Haushalt „wichtiger" sind als ein Kinobesuch oder ein zweites Fernsehgerät. Dass diese gesamtwirtschaftliche Proportionierung der einzelnen Gütergruppen nicht nur vom statistischen Durchschnittseinkommen einer Volkswirtschaft, sondern ganz entscheidend von dessen Verteilung und der Altersstruktur der Bevölkerung - neben anderen Einflussfaktoren - abhängt, bedarf keiner weiteren Erläuterung. Bildungs- und Weiterbildungsdienstleistun- gen werden beispielsweise im Zuge der sozioökonomischen Entwicklung immer wichtiger. Wegen der demografischen Alterung aller altindustrialisierten Gesellschaften wird - wie inzwischen allgemein anerkannt - der Anteil der Gesundheitsund Pflegedienstleistungen steigen.

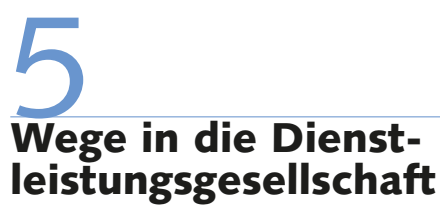

Wie ein konkreter Tertiarisierungsprozess verläuft, hängt von verschiedenen Einflussfaktoren ab. Es steht außer Frage, dass die Wirtschafts- und Gesellschaftspolitik hierbei die Hauptrolle spielt, und es erscheint plausibel, dass die Politik mit der jeweils herrschenden Wirtschaftsideologie eng verwoben ist. Unterschiedliche Tertiarisierungswege korrespondieren mit den unterschiedlichen Formen kapitalistischer Ökonomien. Die Variationsbreite realkapitalistischer Volkswirtschaften (Esping-Anderson 1990) legt die Hypothese nahe, dass die Quantität und vor allem die Qualität der Tertiarisierung einer Volkswirtschaft wesentlich vom spezifischen Typus einer „kapitalistischen Kultur" determiniert werden. Die in den USA verbreitete Staatsphobie zeitigt andere Tertiarisierungstendenzen als das skandinavische solidarstaatliche Verständnis. Und in dem prononciert sozialstaatlichen Deutschland verlief die Tertiarisierung bis Ende der 1970er Jahre ebenfalls anders als in der jüngeren Vergangenheit, in der der Strukturwandel durch eine neoliberalistische Sozialstaatsaversion bestimmt wurde (vgl. auch Lehndorff 2009).

\subsection{DIE „HIGH ROAD“}

Die langfristige Massenarbeitslosigkeit in vielen hoch entwickelten Ländern scheint Fourastiés optimistische Zukunftsvision einer vollbeschäftigten „tertiären Zivilisation" widerlegt zu haben. Dabei wird jedoch übersehen, dass Fourastié und alle, die sich seiner „großen Hoffnung des 20. Jahrhunderts“ angeschlossen haben, voraussetzen, dass die Wirtschafts- und Gesellschaftspolitik den Erfordernissen einer erfolgreichen Tertiarisierung genügen würde. Notwendig wäre einerseits die Ausweitung herkömmlicher qualifizierter Dienstleistungsarbeitsplätze. Zugleich 
ist es erforderlich, dass sich Gesellschaft und Staat rechtzeitig auf quantitativ und qualitativ neue Dienstleistungsbedarfe einstellen, die als Folge der sozialen, der demografischen, der wirtschaftlichen Entwicklung entstehen bzw. aus veränderten Rahmenbedingungen resultieren. Der „Hunger nach Tertiärem“ war von Fourastié durchaus zutreffend prognostiziert worden. Um ihn aber auch angemessen zu befriedigen und gerade dadurch im Dienstleistungsbereich genügend Arbeitsplätze zu schaffen, müssen im Wesentlichen zwei Bedingungen erfüllt sein:

(1) Die Einkommensentwicklung in allen Beschäftigungsbereichen muss sich am gesamtwirtschaftlichen Produktivitätswachstum orientieren, um sowohl die Massenkaufkraft synchron zur gesamtwirtschaftlichen Leistungsfähigkeit zu steigern, als auch der Entstehung von schlecht bezahlter, technologisch rückständiger, prekärer Beschäftigung und der damit einhergehenden gesellschaftlichen Spaltung vorzubeugen.

(2) Eine Ausweitung der materiellen und immateriellen (= personellen) Infrastruktur ist notwendig. Hierbei geht es in der Regel um sehr langfristige orientierte Aufgaben, die für privatwirtschaftliche Akteure entweder aus Renditegesichtspunkten uninteressant sind oder aber bei gewinnwirtschaftlicher Bereitstellung zu krassen Missbräuchen führen können. Beispielsweise können erhebliche Qualitätsmängel auftreten (Beispiel: Rendite versus Patientengesundheit); es kann zur Benachteiligung von Beschäftigten (Niedriglöhne, Arbeitsdruck), von Kunden (Monopolpreise privatisierter Ent- und Versorgungsbetriebe) und der öffentlichen Hände kommen (Sozialisierung bzw. Kommunalisierung von Verlusten bei Public-Private-Partnership-Vereinbarungen).

Unter den heute gegebenen Bedingungen darf ein weiterer Faktor der Dienstleistungsbeschäftigung nicht unerwähnt bleiben: die Frauenerwerbstätigkeit. Vergleichende internationale Studien zeigen eine hohe Korrelation zwischen dem Arbeitsvolumen der Frauen und dem Umfang sozialer und wissensbasierter Dienstleistungen. Aufgrund begrenzter Zeitbudgets sind Haushalte bei einer höheren Zeitverwendung der Frauen für Erwerbsarbeit gezwungen, vermehrt auf soziale Dienstleis- tungen (Kinderbetreuung, Service rund um den Haushalt etc.) zurückzugreifen (sogenanntes „Marketization“) (Bosch 2005, insbes. S. 71). Neben der hierdurch erfolgenden quantitativen Ausweitung der Dienstleistungsbeschäftigung ergeben sich auch qualitative Aspekte: Wenn Haushaltsdienstleistungen vermehrt von professionellen und spezialisierten Dienstleistern erbracht werden, ist sowohl mit einer Steigerung der Qualität der Dienstleistung als auch mit einer Effizienzsteigerung zu rechnen (Schettkat 2010, S. 37).

Das Beispiel der skandinavischen Länder zeigt, dass der Übergang in eine Dienstleistungsgesellschaft mit qualitativ hochwertigen und gut bezahlten Arbeitsplätzen durch staatliche Einflussnahme massiv vorangetrieben werden kann (Bosch/Lehndorff 2005). ${ }^{5}$ Bezogen auf die Bevölkerungszahl müssten in Deutschland nach Berechnungen von Heintze (2010, S. 54) rund 7 Mio. Beschäftigte mehr in öffentlichen Bereichen wie Bildung, Forschung und Entwicklung, Kultur, Gesundheit und Pflege tätig sein, um auf das durchschnittliche Niveau der skandinavischen Länder zu kommen.

\subsection{DIE "LOW ROAD“}

In vielen Ländern mit hohem Pro-KopfEinkommen bewegt sich der Tertiarisierungsprozess jedoch eher in eine andere Richtung. Statt einer Entwicklung hin zu der von Fourastié erhofften ,tertiären Zivilisation" bildeten sich neofeudale Verhältnisse heraus. Privatisierung, Deregulierung und Entstaatlichung gaben einer Entwicklung freien Lauf, die - durchaus vorhersehbar für den Fall der sogenannten Entstaatlichung (Zinn 1978, S. 79ff.) - zu einer im Trend fortschreitenden gesellschaftlichen Spaltung führte. Die Verteilung spricht mehr und mehr jeglicher Leistungsgerechtigkeit Hohn. Abstiegsprozesse überwiegen gegenüber Aufstiegsprozessen. So wird der Mittelstand ausgedünnt und durch die verteilungspolitisch bedingte Schwächung der Massenkaufkraft fehlt die zur Vollbeschäftigung notwendige Nachfrage. Dieser Nachfragemangel lässt das Arbeitsvolumen tendenziell weiter absinken, begünstigt gleichzeitig eine mehr oder weniger starke Deindustrialisierung (etwa in den USA) und führt zu allen möglichen Formen von Billiglohnjobs, speziell im Dienstleistungsbereich. Dieser Irrweg in die Dienstleistungsgesellschaft - von
Fourastié als „tertiäre Krise“ bezeichnet - führt gewissermaßen durch die „Bettlergasse" und droht in eine anhaltende Abwärtsspirale zu münden (Zinn 1993, S. 5ff.; Zinn 1998, S. 109f.).

Inzwischen droht auch die Entwicklung in Deutschland eine solche „low road“ in die Dienstleistungsgesellschaft zu nehmen. Statt einer Ausweitung von öffentlichen und meritorischen Gütern wurden - ideologisch verbrämt mit der Formel vom „schlanken Staat" - öffentliche Beschäftigung und Infrastrukturinvestitionen in einem Umfang abgesenkt, der künftig kaum absehbare Folgekosten und volkswirtschaftliche Leistungseinbußen nach sich ziehen wird. Der Anteil der öffentlich Beschäftigten an der Gesamtbeschäftigung sank nach International Labour Organization (ILO)-Angaben (http://laborsta.ilo.org; Zugriff 21.2.2011) in Deutschland in der Dekade zwischen 1999 und 2009 von ohnehin niedrigem Ausgangsniveau in Höhe von 17,3 \% auf nur noch 14,3\%. Damit gehört Deutschland inzwischen zu den westlichen Ländern mit dem kleinsten öffentlichen Sektor.

\section{Alternativen zur Dienst- leistungsgesellschaft?}

Die empirischen und theoretischen Befunde deuten darauf hin, dass Arbeitsplätze in nennenswertem Umfang zukünftig nur noch im Dienstleistungssektor zu schaffen sind (Bosch/Wagner 2003). Es besteht allgemein Konsens, dass Länder mit hohem Pro-Kopf-Einkommen und alternder Bevölkerung einen starken Bedarfsanstieg an gesellschaftlich notwendigen Dienstleistungen - im Gesundheits-, Pflege-, Erziehungs- wie im Bildungs- und Weiterbildungsbereich ${ }^{6}$ - haben.

\footnotetext{
5 Die Erfolge dieser Politik werden immer wieder bestätigt, zuletzt durch eine international vergleichende Gerechtigkeits-Studie der BertelsmannStiftung (2010, S. 7): „Die nordeuropäischen Staaten sind eine Klasse für sich. Island, Schweden, Dänemark, Norwegen und Finnland führen im Gerechtigkeitsindex mit deutlichem Abstand. Diese Länder erreichen insbesondere sehr gute Werte in den zentralen Gerechtigkeitsdimensionen "Armutsvermeidung " und "Bildungszugang ".

6 Eine Modernisierung des Bildungssektors, d.h. eine deutliche Steigerung des Durchschnittsniveaus, würde auch erhebliche Wertschöpfungspotenziale erschließen. Vgl. Hanushek/Woessmann 2010.
} 
Vor allem aus Sicht kritischer Sozialwissenschaftler und Ökonomen schien es lediglich überkommenen Politikmustern geschuldet, dass die einseitige Förderung des Exports und die damit einhergehende Stärkung des industriellen Sektors bei gleichzeitiger Schwächung der binnenwirtschaftlichen Wachstumspotenziale immer noch im Mittelpunkt wirtschaftspolitischer Anstrengungen steht. Die daraus resultierenden permanenten außenwirtschaftlichen Überschüsse, die eine ständige Verletzung des außenwirtschaftlichen Gleichgewichts sind, führen aber zu erheblichen Verwerfungen in den internationalen Wirtschaftsbeziehungen und behindern in allen beteiligten Volkswirtschaften einen nachhaltigen, effizienten Strukturwandel. Die Defizitländer geraten in Zahlungsbilanzschwierigkeiten, was letztlich auf eine Zahlungsunfähigkeit hinausläuft. Die Überschussländer versäumen es nicht nur, ihr Wachstum mit einem ausgewogenen, die langfristige Stabilität begünstigenden Strukturwandel zu verbinden, sondern sie werden letztlich reale Verluste erleiden. Denn die durch Exportüberschüsse aufgehäuften Devisenreserven drohen sowohl durch inflationäre Entwicklungen im Ausland, vor allem aber infolge von Zahlungsunfähigkeit der Schuldnerländer, real entwertet zu werden. Die globale Krise der Gegenwart, insbesondere aber die Krisenverschärfung im EuroRaum mit seinen de facto am Rande des Bankrotts balancierenden Mitgliedsländern führen die Folgen jener Verwerfungen in den internationalen Wirtschaftsbeziehungen drastisch vor Augen. Die Rückkehr zum außenwirtschaftlichen Gleichgewicht erfordert jedoch keineswegs einen Rückbau der Exportbereiche. Notwendig ist stattdessen die Steigerung der Masseneinkommen und damit der Nachfrage am Binnenmarkt, um über höhere Importe einen Ausgleich der Leistungsbilanz zu erreichen.

Vor dem Hintergrund der vorstehend dargelegten Fakten und Zusammenhänge erscheint eine aktuelle Studie des Bundesministeriums für Wirtschaft und Technologie (BMWi 2010) zur Industriepolitik als eine Übung in strukturpolitischem Anachronismus. Mit der frappierenden Wortschöpfung eines „Systemkopfs der europäischen Wertschöpfungskette" (BMWi 2010, S. 6) wird gewissermaßen einseitig einer Renaissance des industriellen Wachstums das Wort geredet. Dienst- leistungen kommen in der engen Perspektive des Wirtschaftsministeriums lediglich als „unternehmensbezogene Dienstleistungen" und damit nur als Anhängsel der industriellen Entwicklung vor. Die realitätsferne Meinung, Dienstleistungspolitik sei ein quasi „überholtes“ Konzept, ${ }^{7}$ zeugt sowohl von einem analytischen Defizit als auch von großer Blindheit gegenüber den geschilderten beschäftigungs- und wohlstandspolitischen Zukunftserfordernissen, in denen der Expansion von Dienstleistungen eine eigenständige Bedeutung zukommt.

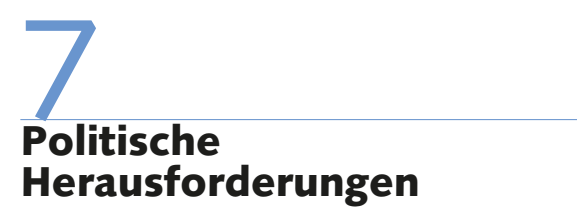

Irgendein Strukturwandel findet in jedem Fall statt. Es kommt aber darauf an, ihn im Interesse der Menschen zu gestalten - im Konfliktfall auch gegen die reinen Gewinninteressen der Shareholder. Es kommen also erhebliche Aufgaben auf die Wirtschafts- und Gesellschaftspolitik zu, und zwar umso mehr, als die schwerwiegenden strukturellen Fehlentwicklungen der Vergangenheit korrigiert werden müssen. Sowohl eine Ausweitung der Staatstätigkeit als auch eine Steigerung der Massenkaufkraft ist unabdingbar, um eine zukunftsweisende Dienstleistungsexpansion zu ermöglichen (Zinn 1993, S. 8ff.).

Ohne ausreichende Masseneinkommen kann sich auch bei vorhandenen großen Bedarfen und einem entsprechenden Angebot keine ausreichende Nachfrage nach Dienstleistungen entfalten. Für Fourastié ergab sich die Kaufkraft quasi naturwüchsig aus dem Rückgang der Preise von Produkten des primären und des sekundären Sektors als Folge des sich dort vollziehenden hohen Produktivitätsfortschritts. Mit dem Sinken der Preise in diesen Sektoren würde - so seine Annahme - Kaufkraft für die Nachfrage nach tertiären Leistungen frei. ${ }^{8}$ Dabei übersah er aber die möglichen und tatsächlich auch eintretenden Verteilungsprobleme. Es gibt nämlich keinen Mechanismus, der dafür sorgt, dass Produktivitäts- und Wachstumsgewinne automatisch bei den Beschäftigten ankommen. Gerade die Entwicklung in Deutschland in der letzten Dekade zeigt, dass vom wirtschaftlichen Wachstum ausschließlich die Gewinn- und Vermögens- einkommensempfänger profitiert haben. Die realen Masseneinkommen stagnieren seit vielen Jahren. Parallel weitete sich der Niedriglohnsektor aus. Nach der jüngsten Wirtschafts- und Finanzkrise scheint sich diese Tendenz ungebrochen fortzusetzen.

Obwohl gerade mehr und vor allem qualitativ hochwertige professionelle Dienstleistungen mit Blick auf die gesellschaftlich wichtigen Zukunftserfordernisse Erziehung, Bildung, Pflege, Gesundheit notwendig wären, verläuft ihre Entwicklung über den Markt völlig unzureichend. Dies liegt weniger an den relativ hohen Dienstleistungspreisen - bekannt als „Baumolsche Kostenkrankheit ${ }^{\text {“9 }}$ - als vielmehr an den unzureichenden Masseneinkommen, die zu niedrig sind, um die notwendigen Dienste kaufen zu können. Durch das Vordringen von Niedriglöhnen, nicht zuletzt auch im Dienstleistungsbereich, wird eine über den Markt laufende Dienstleistungsexpansion stark behindert. Es ergibt sich eine paradoxe Selbstbehinderung des Dienstleistungssektors durch Niedriglöhne. Hieran zeigt sich die verfehlte Strukturpolitik. Der Teufelskreis aus niedrigen Einkommen, einem aus der Not geborenen Dienstleistungsangebot und einer weiteren Ausdehnung unprofessioneller Abfertigung beim Dienstleistungsbedarf zu Niedrigqualitäten und Niedriglöhnen widerspricht allen Merkmalen einer wirklichen „Sozialen Marktwirtschaft" (Zinn 1992).

An dieser Entwicklung trägt die Politik die Hauptschuld. Durch die Arbeitsmarktreformen im Zuge der Umsetzung der Hartz-Gesetze wurde ein enormer Druck auf das Lohnniveau ausgeübt. Leidtragende sind besonders die Beschäftigten im Dienstleistungssektor. Arbeitslose sollten buchstäblich um jeden Preis in einen Job gebracht werden. Dazu wurden Zumutbarkeitsregelungen faktisch außer Kraft gesetzt, Leiharbeit entgrenzt und Billigjobs gefördert. Insofern liegt es auch

\footnotetext{
7 „Viele Experten empfahlen eine Orientierung am angelsächsischen Wirtschaftsmodell. Als Therapie wurde nicht zuletzt eine stärkere Ausrichtung auf den Dienstleistungssektor empfohlen. Heute zeigt sich ein ganz anderes Meinungsbild." (BMWi 2010, S. 6).

8 "Da die landwirtschaftlichen und industriellen Produkte billiger geworden sind, wird das auf diese Weise gesparte Geld in den Tertiärbereich investiert." (Fourastié/Schneider 1989, S. 147.)

9 Von „Krankheit“ zu reden, deutet auf eine ideologisch suggestive Semantik hin. Zu einer anderen Lesart vgl. Bosch/Weinkopf in diesem Heft.
} 
in der Verantwortung der Politik, durch eine Rücknahme dieser Maßnahmen dazu beizutragen, dass die Abkoppelung der Masseneinkommen von der allgemeinen Steigerung des Wohlstands ein Ende hat.

Der Staat muss seine zentrale Führungsaufgabe wahrnehmen, um den Weg in die Dienstleistungsgesellschaft über die „high road“ sicherzustellen: indirekt durch die Schaffung von dienstleistungsfördernden Rahmenbedingungen (Bosch 2005, S. 73; Bosch/Weinkopf in diesem Heft). Hierzu gehören Maßnahmen zur besseren Vereinbarkeit von Beruf und Familie, die Förderung von Qualität und Professionalisierung von Dienstleistungen (etwa durch neue Ausbildungsberufe mit möglichst europaweit anerkannten öffentlich-rechtlichen Abschlüssen $)^{10}$ bis hin zur Unterstützung von Forschung und Entwicklung im industriellen Bereich, etwa hinsichtlich einer effizienteren und ökologischeren Produktion.

Wie die skandinavischen Länder zeigen, muss der Staat aber auch einen direk- ten Einfluss auf den Ausbau des Dienstleistungssektors nehmen. Wirtschafts- und finanzpolitische Maßnahmen müssten diesem Beispiel folgend auf eine Expansion der Beschäftigung im öffentlichen Sektor abzielen. Allerdings würde eine rein quantitative Expansion von Dienstleistungen zu kurz greifen. Erforderlich sind gleichzeitig Maßnahmen zur anhaltenden Qualitätssteigerung des öffentlichen wie des öffentlich gewährleisteten, aber auch des privaten Dienstleistungsangebotes. ${ }^{11}$ Neben einer verstärkten Ausrichtung auf den Bedarf der einkommensschwächeren Schichten, die mehr als wohlhabende Haushalte auf staatliche Förderung und Unterstützung angewiesen sind, ${ }^{12}$ gehört hierzu auch eine klare Orientierung an den Bedürfnissen und Erwartungen der Bürgerinnen und Bürger, um die Zufriedenheit mit öffentlichen Leistungen zu steigern.

Eine Wiederankoppelung der Masseneinkommen an die allgemeine Wohlstandsentwicklung und eine von einer sozialen und ökologischen Steuer- reform (Vereinte Dienstleistungsgewerkschaft 2009; Jarass 2010; Meyer 2010) begleitete Ausweitung der Staatstätigkeit im Allgemeinen und der öffentlichen Beschäftigung im Besonderen wären notwendige Bedingungen für den sukzessiven Übergang in eine, den sozialen und ökologischen Anforderungen gerecht werdende Gesellschaft, in der Dienstleistungen eine immer größere Bedeutung zukommt.
10 Vgl. hierzu den Ansatz, durch „europäische Kernberufe" berufliche Qualifikationen und Kompetenzen länderübergreifend zu definieren, so Wissenschaftlicher Beraterkreis 2008, S. 51ff.

11 Besonders das Ensemble von sozialwirtschaftlichen, kooperativen, bzw. auf Gegenseitigkeit beruhenden Organisationsformen gilt es hier hervorzuheben. Vgl. hierzu Raza 2005, S. $10 f$.

12 Bereits vor geraumer Zeit wurde darauf hingewiesen, dass zwar positive Umverteilungswirkungen öffentlicher Leistungen angenommen werden, eindeutige Belege jedoch fehlen. Vgl. SkarpelisSperk 1978.

\section{LITERATUR}

Bertelsmann-Stiftung (2010) (Hrsg.): Soziale Gerechtigkeit in der OECD - Wo steht Deutschland? Sustainable Governance Indicators 2011, Gütersloh

Bosch, G. (2005): Dienstleistungsbeschäftigung in Deutschland, in: Friedrich Ebert Stiftung (Hrsg.): Das Europäische Sozialmodell im 21. Jahrhundert, Diskussionspapier, Bonn

Bosch, G./Lehndorff, S. (2005): Introduction: service economies - high road or low road?, in: Dies.: Working in the service sector: a tale from different worlds, London, S. 1-31

Bosch, G./Wagner, A. (2003): „Beschäftigungshoffnung Dienstleistungen" braucht politische Begleitung, IAT-Report 4, Gelsenkirchen Bundesministerium für Wirtschaft und Technologie (BMWi) (Hrsg.) (2010): Im Fokus: Industrieland Deutschland. Stärken ausbauen Schwächen beseitigen - Zukunft sichern, Berlin

Clark, C. (1940): The Conditions of Economic Progress, 2. Aufl., London 1951

Esping-Anderson, G. (1990): The Three Worlds of Welfare Capitalism, Cambridge

Fisher, A. (1935): The Clash of Progress and Security, London

Fourastié, J. (1949/1969): Die große Hoffnung des zwanzigsten Jahrhunderts, 2. Aufl., Köln

Fourastié, J. (1967): Gesetze der Wirtschaft von morgen, Düsseldorf/ Wien

Fourastié, J./Schneider, J. (1989): Warum die Preise sinken. Produktivität und Kaufkraft seit dem Mittelalter, Frankfurt a. M./New York

Fuchs, J./Zika, G. (2010): Arbeitsmarktbilanz bis 2025. Demografie gibt die Richtung vor, IAB Kurzbericht 12, Nürnberg
Hanushek, E.A./Woessmann, L. (2010): How Much Do Educational Outcomes Matter in OECD Countries, in: Economic Policy, Fifty-Second Panel Meeting 22-23 October, Centre for Economic Policy Research Heintze, C. (2010): Das skandinavische Vorbild. Zur Rolle des Staates als Arbeitgeber, in: vorgänge 191, 49 (3), S. 50-61

Horn, G./Lindner, F./Niechoj, T./Sturn, S./Tober, S./Truger, A./Will, H. (2011): Herausforderungen für die Wirtschaftspolitik 2011. Der Euroraum in Trümmern?, IMK-Report (59), Januar, Düsseldorf Jarass, L. (2010): Faire und effiziente Steuerpolitik, in: Seidl/Zahrnt (Hrsg.): Postwachstumsgesellschaft. Konzepte für die Zukunft, Marburg, S. 155-166

Joebges, H./Schmalzbauer, A./Zwiener, R. (2009): Der Preis für den Exportweltmeister Deutschland: Reallohnrückgang und geringes Wirtschaftswachstum, IMK Studies (4), Düsseldorf

Lehndorff, S. (2009): Vor der Rückkehr des Staates? Europäische Beschäftigungsmodelle im Umbruch, in: Ders. (Hrsg.): Abriss, Umbau, Renovierung. Studien zum Wandel des deutschen Kapitalismusmodells, Hamburg, S. 197-235

Meyer, B. (2010): Ressourceneffiziente Wirtschaftsentwicklung unter dem Primat ökologischer Ziele, in: Seidl/Zahrnt (Hrsg.): Postwachstumsgesellschaft. Konzepte für die Zukunft, Marburg, S. 167-177 Raza, W. (2005): Jenseits von keynesianischem Wohlfahrtsstaat und liberalem Wettbewerbsstaat? Einführende Überlegungen zur Zukunft öffentlicher Dienstleistungen, in: Kammer für Arbeiter und Angestellte für Wien (Hrsg.): Zur Zukunft öffentlicher Dienstleistungen. Zwischen Staat und Markt - aktuelle Herausforderungen der öffentlichen Dienstleistungserbringung, Wien, S. 1-12 
Reuter, N. (2000): Ökonomik der "Langen Frist“. Zur Evolution der Wachstumsgrundlagen in Industriegesellschaften, Marburg Reuter, N. (2010): Der Arbeitsmarkt im Spannungsfeld von Wachstum, Ökologie und Verteilung, in: Seidl/Zahrnt (Hrsg.): Postwachstumsgesellschaft. Konzepte für die Zukunft, Marburg S. 85-102

Schettkat, R. (2010): Dienstleistungen zwischen Kostenkrankheit und Marketization, Expertise im Auftrag der Friedrich-Ebert-Stiftung, Bonn Seidl, I./Zahrnt, A. (Hrsg.) (2010): Postwachstumsgesellschaft. Konzepte für die Zukunft, Marburg

Skarpelis-Sperk, S. (1978): Wem nützt der Staat? Probleme der schichtenspezifisch ungleichen Inanspruchnahme öffentlicher Leistungen, in: WSI-Mitteilungen 31 (5), S. 262-269

Vereinte Dienstleistungsgewerkschaft (ver.di) (Hrsg.) (2009): Konzept Steuergerechtigkeit, Berlin

Werner, H. (2001): Die Erfahrungen beschäftigungspolitisch erfolgreicher Länder, in: Baethge, M./Wilkens, I. (Hrsg.): Die große Hoffnung für das 21. Jahrhundert. Perspektiven und Strategien für die Entwicklung der Dienstleistungsbeschäftigung, Opladen, S. 245-270
Wissenschaftlicher Beraterkreis der Gewerkschaften IG Metall und der Vereinten Dienstleistungsgewerkschaft ver.di (2008): Berufs-BildungsPerspektiven 2008. Solidarität und gemeinsame Verantwortung, Berlin/ Frankfurt a.M.

Zinn, K. G. (1978): Der Niedergang des Profits. Eine Streitschrift zu den Risiken der kapitalistischen Wirtschaftskrise, Köln

Zinn, K.G. (1992): Soziale Marktwirtschaft. Idee, Entwicklung und Politik der bundesdeutschen Wirtschaftsordnung, Mannheim et al.

Zinn, K.G. (1993): Dienstleistungsgesellschaft oder Krise des tertiären Sektors? Zur quantitativen Analyse der Entwicklung reifer Volkswirtschaften, in: WSI-Mitteilungen 46 (1), S. 1-10

Zinn, K.G. (1998): Von der tertiären Zivilisation in die tertiäre Krise. Zum Verhältnis von Dienstleistungen und Produktion, in: Ders.: Jenseits der Markt-Mythen. Wirtschaftskrisen: Ursachen und Auswege, Hamburg, S. 98-121 